\title{
催化不对称环丙烷化-重排策略发散式构建手性螺缩醛胺和并环缩醛
}

\author{
杨玉环 $a, b$ 徐森苗*,a,b \\ $\left({ }^{a}\right.$ 中国科学院兰州化学物理研究所 兰州 730000) \\ $\left({ }^{b}\right.$ 中国科学院大学 北京 100049)
}

\section{A Versatile Enantioselective Catalytic Cyclopropanation- Rearrangement Approach to the Divergent Construction of Chiral Spiroaminals and Fused Bicyclic Acetals}

\author{
Yang, Yuhuan ${ }^{a, b} \quad \mathrm{Xu}$, Senmiao $^{*, a, b}$ \\ ( ${ }^{a}$ Lanzhou Institute of Chemical Physics, Chinese Academy of Sciences, Lanzhou 730000) \\ $\left({ }^{b}\right.$ University of Chinese Academy of Sciences, Beijing 100049)
}

含有螺缩酮、螺缩醛胺、并环缩醛等结构单元的手 性季碳杂环分子广泛存在于具有重要生物活性的天然 产物、优势手性配体骨架以及功能材料框架体系中(图 1). 比如海洋天然产物 Xyloketal B 具有潜在的治疗帕金 森的应用前景; Azaspiracid-1 则是一种有效的生物毒素. 自从 Ding ${ }^{[1]}$ 和 List 等 ${ }^{[2]}$ 在 2012 年独立报道了首例高对 映选择性分子内螺缩酮化反应以来, 人们发展了许多有 效的方法来构建手性螺缩酮类化合物. 与之形成鲜明对 比的是, 手性的螺缩醛胺和并环缩醛的催化不对称合成 方法非常少见. 虽然通过双金属催化体系 $\mathrm{Au}(\mathrm{I}) / \mathrm{Ni}(\mathrm{II})^{[3]}$ 和 $\mathrm{Au}(\mathrm{I}) /(\mathrm{III})^{[4]}$ 可以有效地合成螺缩酮和螺缩醛胺, 但是 仍然缺少通用和有效的催化不对称方法来发散性地合 成手性螺缩醛胺和并环缩醛.

$\alpha$-重氮酮是一类重要的有机合成子, 在杂原子-氢 插入和环丙烷化等反应中引起了越来越多的关注. 其 中, 铑催化的 $\alpha$-重氮酮与烯醚和烯胺类化合物的环丙烷 化-重排 $(\mathrm{CP}-\mathrm{RA})^{[5]}$ 是构建并环和螺环类化合物的重要 方法(Scheme 1). 然而, 由于该类 CP-RA 过程可能存在 开环的 Zwitterion 中间体, 会导致消旋化问题(Pattern A)，长期以来其催化不对称合成是该领域极具挑战性的 课题. 到目前为止, 只有为数不多的催化剂能够实现该 类不对称催化反应，且存在底物类型少等问题 ${ }^{[6]}$.

近日, 华东师范大学化学与分子工程学院王丽佳课 题组与中国科学院上海有机化学研究所唐勇课题组合 作, 发展了一种基于铜催化的不对称 CP-RA 策略构建
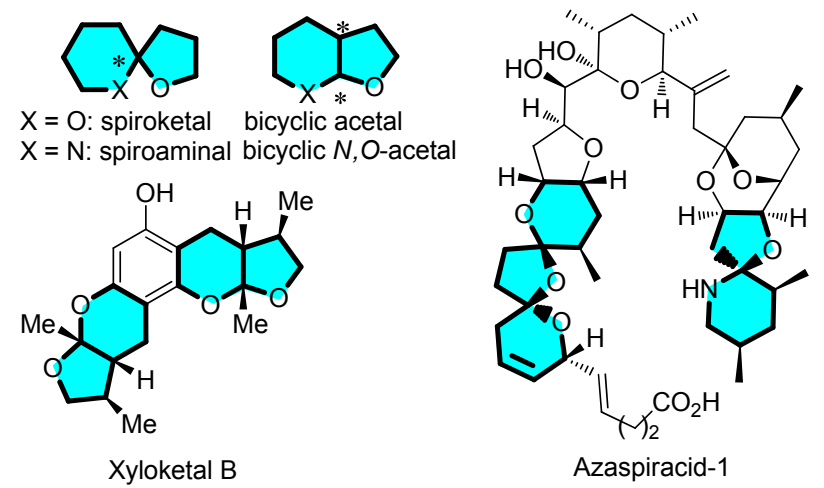

图 1 天然产物中的螺缩醛胺和并环螺缩醛结构单元

Figure 1 Spiroaminal and bicyclic acetal subunits in natural products

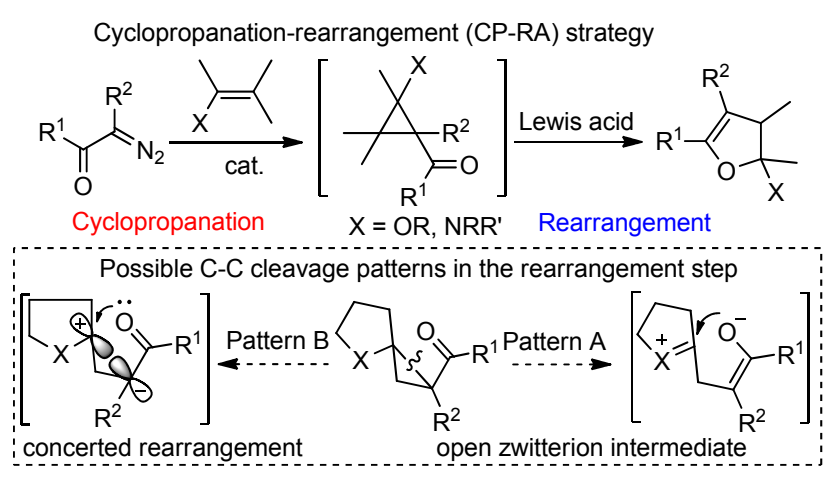

图式 1 CP-RA 方法不对称合成杂双环

Scheme 1 CP-RA approaches for the asymmetric synthesis of heterobicycles

* Corresponding author. E-mail: senmiaoxu@licp.cas.cn. Published online December 6, 2020. 
手性季碳杂环的新方法, 克服了杂原子取代手性环丙烷 重排过程中容易引起消旋化的难题, 实现了手性螺缩醛 胺和并环缩醛/酮的高效多样性合成(Scheme 2) ${ }^{[7]}$.

作者发现, 利用双边臂的手性噁唑啉配体 L1 能够 高效地实现铜催化的环外烯基磺酰胺底物 2 与 $\alpha$-重氮酮 1 的不对称 CP-RA 反应, 生成手性螺缩醛胺 4. 结果表 明, 反应对于五元、六元环状烯基磺酰胺, 含有芳香族 和脂肪族取代基的重氮酩都能得到比较好的结果, 以高 对映选择性得到手性螺缩醛胺产物, 最高 ee 值可达 96\%. 随后，作者利用双边臂的手性啞唑啉配体 L2 实现 了铜催化的环内烯基磺酰胺底物以及烯基醚底物 3 与 $\alpha-$ 重氮酮 1 的不对称 CP-RA 反应, 均能以理想的产率和优 秀的对映选择性获得手性并环缩醛/酮类化合物 $\mathbf{5}$, 最高 $e e$ 值可达 $99 \%$. 值得一提的是, 当作者选用含有环外双 键的四氢吡啶 Boc 酰胺底物 6 与 $\alpha$-重氮酮 1 时, 在配体 L1 存在下能够一步构建同时含有螺缩醛胺和并环缩醛 两个重要手性片段的三环化合物 7, 并取得 90/10 $d r$ 和 99\% ee.

作者也对该反应的对映选择性控制进行了初步研 究. 经过一系列对比实验, 结合核磁原位跟踪手段, 揭 示了决定反应对映选择性的可能途径. 该工作实现了手 性季碳杂环的高效构建, 铜催化的 CP-RA 策略首次被 成功应用于不对称合成手性螺缩醛胺和并环缩醛/酮中. 该方法为快速构建具有复杂环系、结构多样性的手性杂 环化合物提供了简洁高效的新方法.

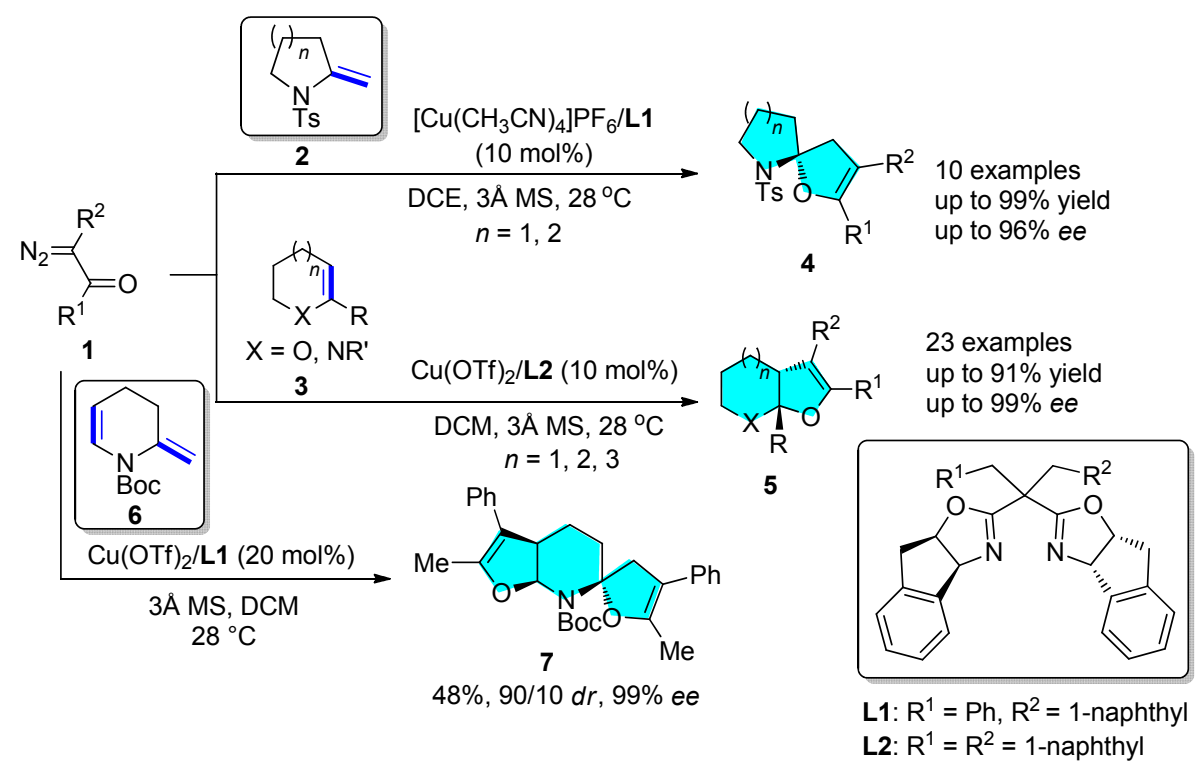

图式 2 铜催化的不对称 CP-RA 反应

Scheme 2 Copper-catalyzed asymmetric CP-RA reactions

\section{References}

[1] Wang, X.; Han, Z.; Wang, Z.; Ding, K. Angew. Chem., Int. Ed. 2012, 51, 936 .

[2] Coric, I.; List, B. Nature 2012, 483, 315.

[3] J. Li, L. Lin, B. Hu, X. Lian, G. Wang, X. Liu, X. Feng, Angew. Chem., Int. Ed. 2016, 55, 6075.
[4] Gong, J.; Wan, Q.; Kang, Q. Adv. Synth. Catal. 2018, 360, 4031.

[5] Pirrung, M. C.; Zhang, J. C.; Mcphail, A. T. J. Org. Chem. 1991, $56,6269$.

[6] Müller, P.; Bernardinelli, G.; Allenbach, Y. F.; Ferri, M.; Grass, S. Synlett 2005, 1397.

[7] Zhou, L.; Yan, W.-G.; Sun, X.-L.; Wang, L.; Tang, Y. Angew. Chem., Int. Ed. 2020, 59, 18964. 